\title{
Tipicidad de identidad de género y comparación intergrupal
}

\author{
MerCedes López SÁez \\ Universidad Nacional de Educación a Distancia
}

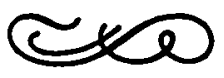

\section{Resumen}

El objetivo de esta investigación era explorat, en grupos extremos en tipicidad de género, los niveles medio y subordinado sobre los que, según la Teoría de la Categorización del Yo de Tumer y col., se construye el autoconcepto. Se ban tomado dos grupos de sujetos: aquellos que establecen su identidad de género según los patrones típicos culturales (mujeres femeninas y bombres masculinos) y aquellos que la establecen con patrones culturalmente atípicos (mujeres masculinas y bombres femeninos). La bipótesis general de la que partía el trabajo ha sido que no existirian diferencias entre estos dos grupos en el grado de identificación con su endogrupo ni en la valoración de él, sino en la representación que tienen de la dicotomia hombre-mujer. Mediante un diseño factorial $2 \times 2$ (tipo de identidad: típica o atípica y sexo) se han analizado las siguientes variables dependientes: estereotipia de género, bomogeneidad del endogrupo y del exogrupo, evaluación del endogrupo y del exogrupo, semejanza al endogrupo y al exogrupo. Los resultados confirman la bipótesis general. Se han encontrado diferencias entre los grupos con identidad típica y atípica sólo en estereotipia de género y en homogeneidad del endogrupo y del exogrupo. También se ba encontrado que existe relación entre estas tres variables.

Palabras clave: Endogrupo, Exogrupo, Esterotipia, Homogeneidad.

\section{Typicity of gender identity and intergroup comparison}

\section{Abstract}

This research focused on groups extreme in gender typicity, and its main aim was to explore the intermediate and subordinate levels upon which self-concept is built, according to Self-Categorization Theory. Two groups were created: 1) the first consisted of those subjects whose gender identity followed the typical cultural pattem (i.e., feminine females and masculine males); 2) the second comprised the atypical ones (i.e., masculine females and feminine males). Our general bypotbesis predicted differences between these two groups in the representation of the male-female dichotomy, but not in the degree of identification with the ingroup or ingroup evaluation. A $2 \times 2$ factorial design (typicallatypical and sex) was used. The dependent variables considered were as follows: gender stereotyping, ingroup and outgroup bomogeneity, ingroup and outgroup evaluation and similarity between ingroup and outgroup. The results confirmed the general bypotbesis. Differences between groups appeared only in the gender stereotyping and ingroup and outgroup homogeneity. We also found relationships among these three variables.

Key words: Ingroup, Outgroup, Stereotype, Homogeneity.

Dinección de la autora: Facultad de Psicología. Uned, Ciudad Universitaria 28040 Madrid. 
Un importante número de investigaciones han tratado de comprobar, en las dos últimas décadas, la influencia de la identificación con los roles de género sobre la conducta de estereotipia sexual. Los resultados obtenidos en estas investigaciones, aunque no permiten llegar a conclusiones definitivas e inequívocas sobre las leyes que regulan esta relación, sí han puesto de manifiesto la necesidad de considerar la identidad de género de los sujetos como variable moduladora en los procesos cognitivos relacionados con las categorías hombre y mujer.

Se tiende a diferenciar entre sexo y género, utilizando este último término para referirse a las características no biológicas (rasgos y roles) que se asignan diferencialmente a hombres y mujeres. El género es, entre otras cosas, un esquema para la categorización social de los individuos (Sherif, 1982). La identidad del rol de género se refiere, por tanto, a la relación psicológica del individuo con esos esquemas asociados a cada una de estas dos categorías. Esta relación implica tanto el conocimiento por parte del individuo del esquema de categorías para el género, como reacciones valorativas de aceptación y rechazo de ellas (Moya, 1985).

La categorización hombre-mujer parte de un dimorfismo sexual claro. Sobre estas diferencias la mayoría de las culturas han establecido diferentes roles para cada sexo, no sólo distintos sino en muchos casos antagónicos. Siguiendo la revisión de Deaux (1985), en un estudio realizado sobre 30 países, Williams y Best (1982) encontraron un gran consenso transcultural al asignar rasgos instrumentales y agentes a los varones (agresividad, independencia, competitividad) y rasgos expresivos y comunales a las mujeres (afectividad, dependencia, preocupación por los demás). No obstante, en otras investigaciones en que se compararon grupos dentro de una misma cultura se han encontrado diferencias entre grupos que asumían distintos valores o pertenecían a diferentes religiones.

También dentro de cada grupo social (por ejemplo, grupos de distintas edades u ocupaciones) se supone que existen diferencias individuales en los esquemas de género, fruto de procesos de socialización autobiográficos que han tenido lugar a través de interacciones recíprocas entre el individuo y su medio social más inmediato (Gracia, Musitu, Escarti, 1988). Estas diferencias se mantienen tanto al aplicar estos esquemas a otras personas como al aplicarlos a uno mismo.

Dentro de esta línea de investigación de diferenciación individual en esquemas de género, los instrumentos de medida más utilizados han sido el Bem Sex Role Inventary (BSRI) de Bem (1974) y el Personal Attributes Questionnaire (PAQ) de Spence, Helmreich y Stapp (1974). Ambos cuestionarios constan de dos escalas, una de Feminidad y otra de Masculinidad, similares en contenido y propiedades psicométricas, habiéndose encontrado altas correlaciones entre las escalas paralelas de los dos instrumentos (Edwards y Spence, 1987). Estas escalas seleccionan rasgos culturalmente más asociados a las mujeres que a los hombres (ítems femeninos) o más asociados a los hombres que a las mujeres (ítems masculinos). En función de la puntuación en masculinidad y feminidad respecto a la mediana del grupo, los sujetos pueden clasificarse en: femeninos (altos en $\mathrm{F}$ y bajos en $\mathrm{M}$ ), masculinos (altos en $\mathrm{M}$ y bajos en $\mathrm{F}$ ), andróginos (altos en $\mathrm{M}$ y $\mathrm{F}$ ) $\mathrm{e}$ indiferenciados (bajos en $\mathrm{M}$ y $\mathrm{F}$ ).

\section{Teorías de los esquemas de género}

Las teorías en torno a la relación entre rasgos de personalidad femeninos o masculinos y conductas estereotipadas de género se han desarrollado funda- 
mentalmente dentro del marco más genérico de las teorías de los esquemas.

En Psicología el término esquema se utiliza para referirse a estructuras de conocimiento preexistentes en la mente del sujeto, relativas a un tema o área concreta y organizadas a partir de la experiencia previa. Los esquemas guían el procesamiento de la información e influyen en la conducta.

Los esquemas sociales son aquellos relacionados con el conocimiento que tenemos sobre las personas y sus interacciones. Dentro de estos esquemas, los autoesquemas, o conocimiento que la persona tiene de sí mismo, son especialmente importantes.

La génesis de un esquema cognitivo no es un proceso estrictamente individual, sino que tiene una dimensión sociocultural. Por ello, podemos considerar los esquemas de personas sociales en un doble sentido, por su origen y por referirse a una categoría social (De Vega, 1984).

El esquema genérico de una determinada categoría social es el conjunto de rasgos, motivaciones, roles y conductas que caracterizan a los miembros prototípicos de esa categoría y los diferencia de los miembros de categorías excluyentes. La pertenencia a una determinada categoría supone semejanza y diferencias en unas dimensiones específicas. Esas semejanzas y diferencias entre miembros de categorías constituyen un conjunto de creencias social y culturalmente aceptadas.

Los estereotipos son estos esquemas socialmente compartidos que organizan el conocimiento en torno a una categoría social, pudiendo considerarse como los esquemas de rol más genéricos (Páez, Villarreal, Etxeberria, Valencia, 1987). Los estereotipos de hombres y mujeres incluirían todas las características, tanto físicas como psicológicas, culturalmente aceptadas sobre lo que es prototípico de esas dos categorías. Al hablar de estereotipia de género nos referimos, exclusivamente, a las características psicosociales que se consideran prototípicas de estas dos categorías excluyentes.

La teoría del esquema de género de Bem (1981) propone que el autoconcepto de género deriva del esquema de género del individuo, es decir, del esquema de roles asociados a varones y mujeres. El niño aprende el contenido social de estos esquemas, los atributos asociados a su sexo y por tanto a él mismo. El autoconcepto de género sería una parte de esos esquemas más generales. Esta teoría se centra no en el contenido de estos esquemas sino en la influencia que ejercen sobre el procesamiento de la información relacionada con el género, incluso en el procesamiento de la información sobre sí mismo.

Esta teoría predice que los individuos que se identifican con el rol de género tradicional de su sexo, es decir, las mujeres femeninas y los hombres masculinos, difieren del resto en que poseen una mayor facilidad para procesar la información en general, y también el conocimiento de sí mismos, de acuerdo con los esquemas de género que dicotomizan lo masculino y lo femenino. Solamente estos sujetos sexo-tipificados serían esquemáticos al procesar información sobre el género.

La teoría propuesta por Markus y colaboradores (1982), sobre la relación entre autoesquema de género y el procesamiento de información sobre este tema, es de un cognitivismo aún más estricto que la teoría de Bem. Para estos autores los sujetos masculinos según la tipología de Bem son esquemáticos al procesar información relacionada con lo masculino y no con la información asociada a lo femenino, los sujetos femeninos son esquemáticos sólo respecto a la información relacionada con lo femenino, los andróginos lo son respecto a am- 
bos tipos de información y únicamente los indiferenciados no serían esquemáticos respecto al género. Según esta teoría el sexo de los individuos no influiría en el procesamiento de la información.

De ambas aproximaciones se derivan distintas predicciones de conductas estereotipadas respecto al género. Para Bem, los sujetos sexotipificados diferirían del resto, es decir, de los andróginos, indiferenciados y de los sujetos con identidad de rol de género cruzada o atípica (hombres femeninos y mujeres masculinas). Para Markus y colaboradores las predicciones dependerán del tipo de estímulo: ante un estímulo masculino los sujetos altamente identificados con lo masculino (masculinos y andróginos) se comportarán igual y distinto de los femeninos e indiferenciados; simétricamente, si el estímulo es femenino los sujetos con alta identidad femenina (femeninos y andróginos) tendrán el mismo comportamiento entre sí y distinto del resto.

No obstante, la revisión de las investigaciones que se han centrado en la puntuación en las escalas de feminidad y masculinidad como predictores no apoyan, claramente, ninguno de los dos modelos ni han podido replicarse, de forma inequívoca, los trabajos empíricos con los que se han demostrado estas dos teorías. Por ejemplo, los resultados de Taylor y Falcone (1982) no coinciden con los hallados, con diseños similares, por Frable y Bem (1985) o por Beauvais y Spence (1987). Tampoco los de Deaux, Kite y Lewis (1985) reproducen los de Bem (1981) al replicar esta investigación. Payne, Connor y Colletti (1987) comparan el modelo de Bem y el de Markus utilizando dos tipos de variables dependientes: reconocimiento por parte de los sujetos de una serie de características extraídas del PAQ como propias y recuerdo de una lista de nombres idéntica a la utilizada por Bem (1981). En la tarea de memoria no se confirman las predicciones de ninguna de las dos teorías, encontrando sólo una confirmación parcial del modelo de Markus en la tarea de reconocimiento de características. Edwards y Spence (1987) también comprueban la validez de los modelos de Bem y Markus no encontrando apoyo empírico para ninguno de los dos.

A la vista de estos resultados, Spence (1985) propone un nuevo modelo de análisis de los procesos relacionados con el género que tenga en cuenta los múltiples factores asociados a este concepto. Para esta autora, no está justificada la utilización del BSRI y el PAQ para medir constructos tan abstractos como masculinidad y feminidad, tipificación sexual, esquematización de género o identidad de rol sexual. Estos cuestionarios de personalidad miden sólo dos constelaciones de rasgos, por un lado rasgos expresivos o comunales y por otro rasgos instrumentales o agentes. Las puntuaciones en estos instrumentos pueden resultar útiles para predecir comportamientos relacionados con, o que puedan verse afectados por, estos dos tipos de rasgos, pero no en otros casos.

Spence critica las teorías sostenidas por Bem y Markus por asumir que todos los fenómenos que tienen que ver con el género están relacionados entre sí formando un conjunto de procesos comunes y monolíticos. Hay muchas dimensiones relacionadas con el género como pueden ser las características asociadas a hombres y mujeres, actitudes más o menos convencionales respecto a los roles sexuales, saliencia del género al procesar información, etc. El modelo multifactorial propuesto por Spence destaca la necesidad de medir independientemente cada una de estas dimensiones, y no mediante un tipo de instrumento único como pueden ser el BSRI o el PAQ. También subraya esta autora el hecho de que a pesar de que pocos hombres y mujeres se identifican con los estereotipos de genero presentes en nuestra sociedad, salvo raras excepciones como en 
el caso de los transexuales, todos son considerados por los otros y por sí mismos como miembros de las categorías sexuales a las que pertenecen. Es decir, la identidad social y personal de los individuos como hombres y mujeres se mantiene a pesar del nivel de aceptación de los roles tradicionales, que es lo que miden los inventarios de identidad de rol de género.

A pesar de las críticas que puedan hacerse a cómo se miden las diferencias individuales en cada una de estas dimensiones, lo que no se pone en cuestión es la utilidad de las diferencias personales en los fenómenos relacionados con el género como predictores de diferencias en percepción y comportamiento de las categorías hombre y mujer. Como se pregunta Frable (1989), ¿no tienen nada que ver las características personales de género con actitudes, valores, preferencias, roles y comportamientos de género?, o, formulando esta pregunta en otros términos, ¿existe relación entre la identidad personal de género y la representación que el individuo tiene de los hombres y las mujeres? Porque el sentido común nos dice que sí están relacionados, sigue teniendo interés el seguir explorando en esta área.

\section{Identidad Personal e Identidad Social: La teoría de la categorización del yo}

El estudio que aquí se presenta trata de determinar la relación entre identidad de rol de género (medida con el BSRI) y una serie de medidas que emergen de la comparación hombre y mujer: estereotipia, favoritismo y homogeneidad. También se ha tratado de determinar la relación del sujeto con su propia categoría sexual y con la otra categoría mediante una medida de semejanza con esas categorías.

En el enfoque de este trabajo se han tenido en cuenta algunos de los supuestos propuestos por Turner y colaboradores (1987) en su teoría de la categorización del yo. En esta teoría, el proceso de comparación social se considera esencial para establecer la identidad personal. La identidad personal o autoconcepto estaría constituida por el conjunto de representaciones cognitivas de sí mismo de que dispone una persona. $\mathrm{El}$ autoconcepto incluye componentes muy diversos, altamente diferenciados, que funcionan con relativa independencia y que constituyen distintas representaciones del yo. Esas representaciones cognitivas del yo adoptan la forma de categorizaciones del yo y, como todas las categorías, se basan en la percepción de semejanzas intraclase y diferencias interclase entre estímulos. La categorización del yo se establece, al menos, en tres niveles jerárquicos: un nivel superordenado en el que la categorización del yo se basa en la pertenencia a la especie humana y en las características compartidas por todos los humanos frente a otras especies; un nivel medio, basado en la comparación entre grupos sociales y que define a la persona como perteneciente a unos grupos y no a otros (categorizaciones endogrupo-exogrupo); y un nivel subordinado, basado en la comparación de uno mismo con el resto de miembros del propio grupo, que le define como persona única.

El estudio se ha centrado en dos tipos de sujetos: aquellos que establecen su identidad de género según los patrones típicos culturales (mujeres femeninas y hombres masculinos) y aquellos que la establecen con patrones culturalmente atípicos (mujeres masculinas y hombres femeninos). El objetivo era explorar, en estos grupos extremos en tipicidad de identidad de género, los niveles medio y subordinado sobre los que, según la teoría de la categorización del yo, se construye el autoconcepto. 


\section{4}

A pesar de que el objetivo del estudio era fundamentalmente exploratorio, se ha partido de la hipótesis de que los sujetos con identidad de género atípica no difieren de los sujetos con identidad típica en el grado de identificación con su endogrupo ni en la valoración que tienen de él, sino en la representación que tienen de la dicotomía hombre y mujer. Es decir, los sujetos con identidad de género típica y atípica construirían su identidad personal con procesos similares de comparación endogrupo-exogrupo.

De esta hipótesis general se derivan las siguientes hipótesis concretas:

Primera hipótesis: Los sujetos con identidad de género típica tendrán una representación de las categorías hombre y mujer más estereotipada que los de identidad atípica.

Segunda hipótesis: los sujetos con identidad de género típica y atípica no diferirían en la percepción de homogeneidad del endogrupo y el exogrupo. Ambos percibirían al exogrupo como más homogéneo que al endogrupo.

Tercera hipótesis: los sujetos con identidad de género típica y atípica valorarán más el endogrupo que al exogrupo.

Cuarta hipótesis: los sujetos con identidad de género típica y atípica no diferiran en semejanza con los miembros de su propia categoría sexual (endogrupo) al compararse con ellos.

Quinta hipótesis: los sujetos con identidad de género típica y atípica no diferiran en semejanza con los miembros de la otra categoría sexual (exogrupo) al compararse con ellos.

Sexta hipótesis: tanto los sujetos con identidad de género típica como los de identidad atípica se considerarían más semejantes al endogrupo que al exogrupo.

\section{Muestra}

La muestra la componían 138 alumnos de COU de un centro público de Madrid, de los cuales 89 eran chicas y 49 chicos, con edades comprendidas entre los 16 y 19 años. El $75 \%$ de la muestra siempre había asistido a un centro mixto. Siguiendo la tipología de Bem, la distribución de los sujetos era la siguiente: $28 \%$ masculinos ( 16 chicas y 18 chicos), $29 \%$ femeninos ( 30 chicas y 5 chicos), $23 \%$ andróginos ( 20 chicas y 8 chicos) y $20 \%$ indiferenciados ( 15 chicas y 9 chicos). Por tanto, el grupo de sujetos con identidad típica lo formaban las 30 chicas «femeninas» y los 18 chicos «masculinos» (35\% de la muestra). y el de identidad atípica las 16 chicas «masculinas» y los 5 chicos «femeninos» (15\% de la muestra).

\section{Instrumento y procedimiento}

Los sujetos contestaron a los 60 ítems que componen el Inventario de Roles Sexuales de Bem aplicados a las mujeres en general, a los hombres en general y a ellos mismos. Los sujetos respondían en qué medida cada uno de los adjetivos del cuestionario servía para describirse a sí mismo, a los hombres y a las mujeres. En todos los casos la puntuación iba de 1 «nunca tienen esa característica» a 7 «siempre tienen esa característica». El orden de presentación de los tres cuestionarios fue en primer lugar el que correspondía a la categoría sexual del sujeto, es decir, el aplicado a las mujeres en el caso de las chicas y el aplicado a los hombres en el caso de los chicos, en segundo lugar el correspondiente al 
sexo opuesto, y en tercer lugar el aplicado a sí mismos. Se recogía cada cuestionario antes de entregar el siguiente.

\section{Diseño}

Se ha utilizado un diseño $2 \times 2$ en el que se consideraron como variables independientes el sexo y la tipicidad de la identidad de género dicotomizada en dos niveles: típicos (chicas que puntuaban por encima de la mediana en feminidad y por debajo en masculinidad, y chicos que puntuaban por encima de la mediana en masculinidad y por debajo en feminidad) y atípicos (chicas que puntuaban por encima de la mediana en masculinidad y por debajo en feminidad y chicos que puntuaban por encima de la mediana en feminidad y por debajo en masculinidad). Como variables dependientes se han utilizado las siguientes medias:

1) Estereotipia de género. Para cada uno de los rasgos femeninos y masculinos que componen la escala se ha hallado una medida de estereotipia basada en la razón entre la puntuación otorgada por el sujeto a los hombres y a las mujeres (Martin, 1987). En el caso de ítems masculinos, el numerador de la razón lo constituía la puntuación aplicada a los hombres y el denominador la puntuación aplicada a las mujeres. Para los ítems femeninos la razón se invertía, de forma que el numerador lo constituía la puntuación de mujeres y el denominador la de hombres. Esta medida comparativa permite establecer que a mayor puntuación, los sujetos estereotipan más a hombres y mujeres en la dirección tradicional. La medida total de estereotipia se ha hallado computando la suma de las medidas de razón de cada ítem.

2) Homogeneidad del endogrupo. Cuantificada por el número de veces que el sujeto utilizaba los extremos de la escala, siempre o nunca, para describir a su categoría sexual en los 60 ítems del cuestionario.

3) Homogeneidad del exogrupo. Operacionalizada como en la variable anterior pero referida a la categoría sexual distinta a la propia.

4) Evaluación del endogrupo. Suma de las 10 puntuaciones positivas y neutras contenidas en el BSRI de la que se restaban las puntuaciones en los 10 ítems negativos y neutros, aplicadas a la categoría sexual a la que pertenecía el sujeto.

5) Evaluación del exogrupo. Operacionalizada como en la variable anterior pero referida a la categoría sexual distinta de la del sujeto.

6) Semejanza con la propia categoría (endrogrupo) en rasgos femeninos y masculinos. Para cada uno de los rasgos femeninos y masculinos que componen la escala se ha hallado una medida de semejanza basada en la razón entre la puntuación otorgada por el sujeto a los miembros de su categoría y la que se adjudica a sí mismo. Es decir, en el numerador se coloca la puntuación que da a los hombres si es un chico y la que da a las mujeres si es una chica y en el denominador su propia puntuación. La puntuación total se ha computado como suma de las medidas de razón de cada tipo de ítems.

7) Semejanza con la otra categoría (exogrupo) en rasgos femeninos y masculinos. Se ha operacionalizado como en la variable anterior pero situando en el numerador la puntuación otorgada a la categoría sexual distinta de la propia.

\section{Resultados}

En relación con la primera de las hipótesis, los resultados indican que los sujetos con identidad de género típica tienen una percepción más estereotipada 
de las categorías hombre y mujer que los sujetos con identidad de género atípica ( $\bar{x}=61,6$ y $\bar{x}=59,8$, respectivamente), aunque esta diferencia es sólo tendencialmente significativa $(F=2,9 ; P=0,09)$. No hay influencia del sexo de los sujetos sobre la medida de estereotipia, ni tampoco interacción del sexo con el tipo de identidad.

Los resultados no confirman la segunda hipótesis. El grupo de sujetos con identidad de género típica tiene una percepción del endogrupo más homogénea $(\bar{x}=6,43)$ que el grupo de sujetos con identidad de género atípica $(\bar{x}=3,66)$ $(F=3,10 ; P=0,08)$. Estos resultados se repiten en la percepción de homogeneidad del exogrupo, donde también el grupo de identidad de género típica supera al grupo de identidad de género atípica $(\bar{x}=6,9$ y $\bar{x}=3,8$, respectivamente), $(F=3,50 ; P=0,06)$. La homogeneidad percibida en el endogrupo no se diferencia de la percibida en el exogrupo ni en el grupo de identidad típica ni en el de atípica $(\mathrm{t}=-.57 ; \mathrm{P}=.57$ para los primeros y $\mathrm{t}=-.20 ; \mathrm{P}=.84$ para los segundos). La influencia del sexo o la interacción sexo por tipo de identidad de género sobre la homogeneidad del endogrupo o del exogrupo no son significativas.

A la vista de estos resultados, se ha comprobado si existía relación entre estereotipia, percepción de homogeneidad del endogrupo y percepción de homogeneidad del exogrupo, en el conjunto de la muestra. Esta relación entre las tres variables se ha confirmado. El coeficiente de correlación entre estereotipia y percepción de homogeneidad del endogrupo es de $0,42(\mathrm{P}<0,001)$; entre estereotipia y percepción de homogeneidad del exogrupo el coeficiente de correlación es de $0,27(\mathrm{P}<0,01)$; y entre percepción de homogeneidad del endogrupo y el exogrupo el coeficiente de correlación es $0,70(\mathrm{P}<0.001)$.

Los datos obtenidos en la evaluación del endogrupo y el exogrupo confirman la tercera hipótesis. Los sujetos de identidad típica valoran más al endogrupo $(\overline{\mathrm{x}}=3.41)$ que al exogrupo $(\overline{\mathrm{x}}=-.31) ;(\mathrm{t}=2,35 ; \mathrm{P}=0,02)$. También los sujetos de identidad atípica valoran más al endogrupo $(\bar{x}=4,83)$ que al exogrupo $(\bar{x}=-2,50)(t=2,34 ; P=0,03)$.

También se ha analizado la influencia de las variables sexo y tipo de identidad sobre la evaluación del endogrupo y del exogrupo. En la evaluación del endogrupo sólo es significativo el efecto de la variable sexo. Las chicas evalúan más positivamente a su endogrupo $(\bar{x}=7)$, es decir, a las mujeres, que los chi$\cos (\bar{x}=-1.36)$ a los hombres $(F=11,52 ; P=0.001)$. En la evaluación del exogrupo no hay influencia de las variables sexo y tipo de identidad separadamente, aunque sí es significativa la interacción $(\mathrm{F}=15,48 ; \mathrm{P}=0,000)$. Entre las chicas, las de identidad típica valoran más al exogrupo, es decir, a los hombres, que las de identidad atípica. Entre los chicos son los de identidad típica los que menos valoran al exogrupo, es decir, a las mujeres.

La cuarta y quinta hipótesis también se han confirmado. El tipo de identidad de género no influye en la semejanza con el endogrupo ni con el exogrupo. Tampoco influye el sexo de los sujetos. Sin embargo, sí es significativa la interacción del sexo y el tipo de identidad sobre la semejanza al endogrupo $(\mathrm{F}=15,48$; $P=0,00)$ y sobre la semejanza al exogrupo $(F=15,99 ; P=0,00)$. En semejanza al endogrupo, entre las mujeres, son las de identidad atípica las que más se asemejan a su grupo (las otras mujeres); entre los hombres, son los de identidad típica los que más se asemejan a los otros hombres. En semejanza al exogrupo, las mujeres de identidad atípica se consideran más semejantes a los hombres que las de identidad típica, invirtiéndose este patrón para los hombres, entre 
los que se consideran más semejantes a las mujeres los chicos de identidad típica que los de atípica. Para interpretar mejor estas interacciones, se ha diferenciado en la semejanza al endogrupo y al exogrupo entre rasgos femeninos y masculinos.

Según están operacionalizadas estas variables, la puntuación 20 indicaría que el sujeto se considera igual a la categoría de comparación (endogrupo o exogrupo) en ese tipo de rasgos. Puntuaciones inferiores a 20 indican que considera que la categoría con la que se compara posee en menor medida que él ese tipo de rasgos. Puntuaciones superiores a 20 indican que considera que la categoría con la que se compara posee en mayor medida que él ese tipo de rasgos. En los gráficos 1, 2, 3 y 4 se representa la distancia al punto de igualdad de la categoría (20) de los cuatro grupos en estudio.
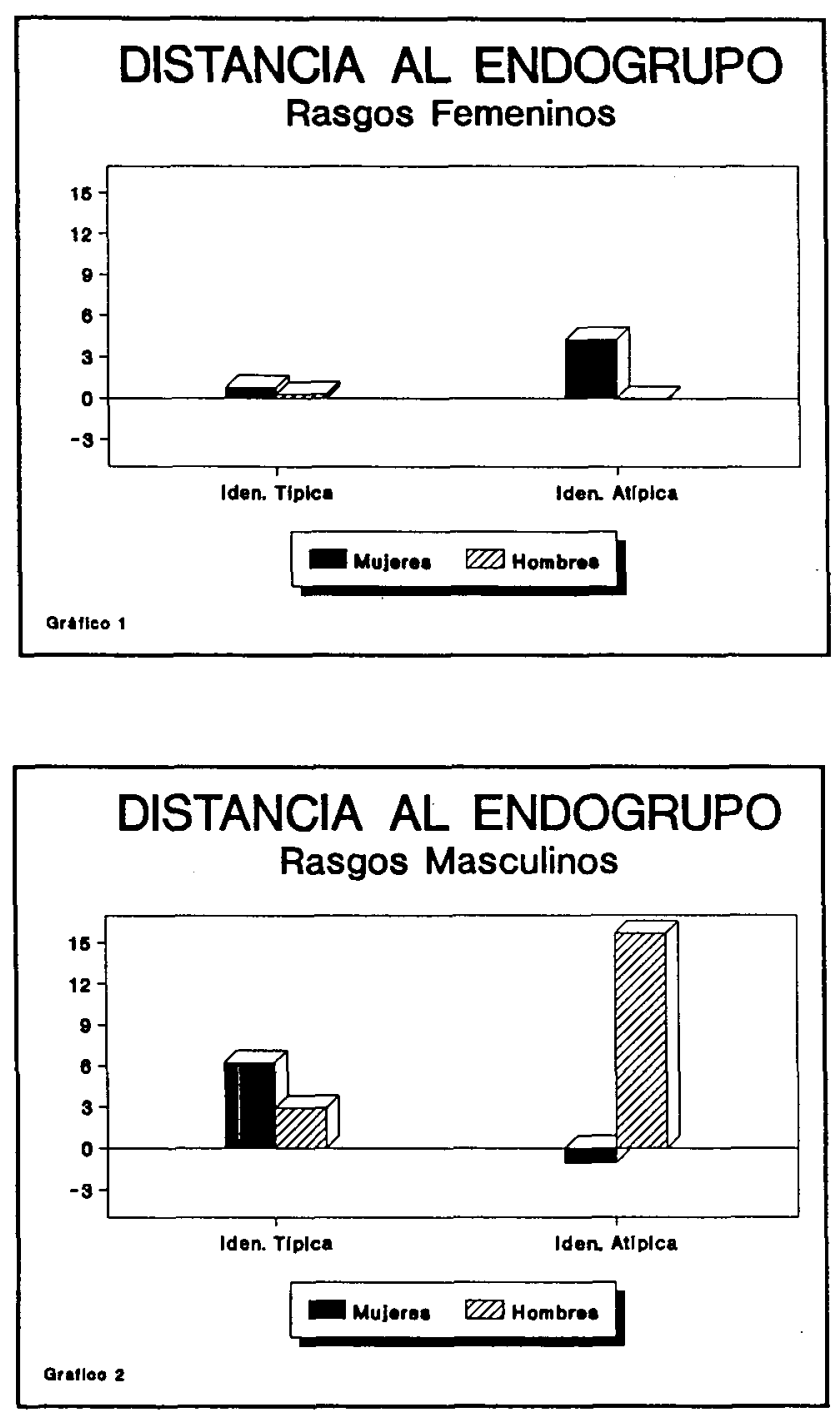

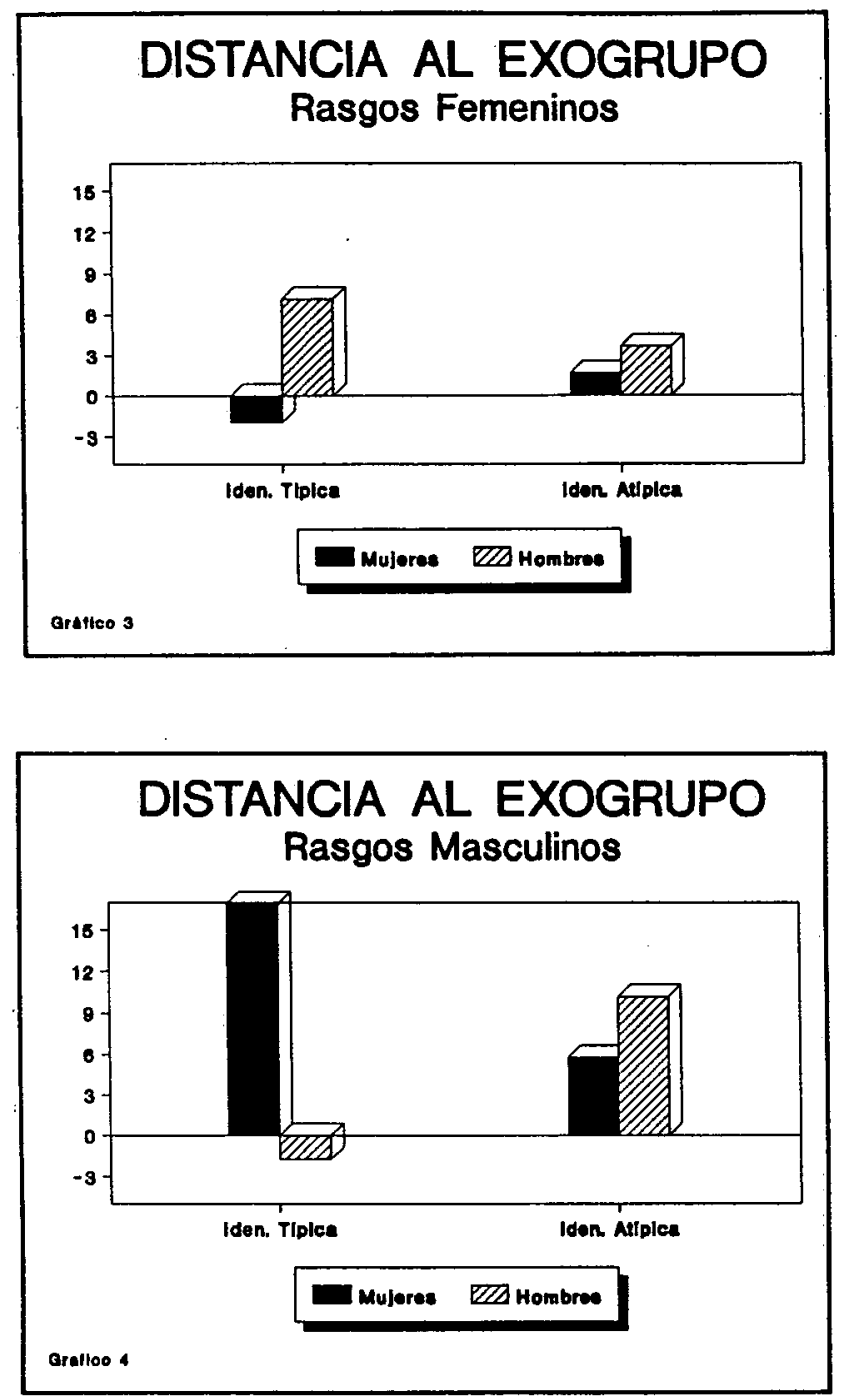

Como puede observarse en estos gráficos, las mujeres con identidad de género femenina consideran que las otras mujeres están muy igualadas en rasgos femeninos a ellas $(0,77)$, pero que en rasgos masculinos, las otras mujeres las superan $(6,22)$; en la comparación con los hombres, en rasgos femeninos consideran que éstos poseen menos características de este tipo que ellas ( -2$)$, y que en rasgos masculinos ellos las superan ampliamente $(16,85)$.

Los hombres con identidad de género masculina consideran a los otros hombres iguales a ellos en rasgos femeninos $(0,32)$ y algo superiores en rasgos masculinos $(2,94)$; al compararse con las mujeres, consideran que éstas tienen más rasgos femeninos que ellos $(7,12)$ y menos masculinos $(-1,75)$.

Las mujeres con identidad de género masculina consideran que las otras mujeres tienen más rasgos femeninos que ellas $(4,3)$ pero menos masculinos $(-1,11)$; respecto a los hombres, consideran que éstos tienen más rasgos femeninos que ellas $(1,66)$ y también más rasgos masculinos $(5,7)$. 
Los hombres con identidad de género femenina se consideran iguales a los otros hombres en rasgos femeninos, pero que éstos les superan ampliamente en rasgos masculinos $(15,7)$; comparándose con las mujeres consideran que éstas les superan en rasgos femeninos $(3,64)$ y más aún en rasgos masculinos $(10,2)$.

En cuanto a la sexta hipótesis, se ha confirmado para el grupo de sujetos con identidad típica $(t=-6,55 ; \mathrm{P}=0.001)$ y tendencialmente para el grupo de identidad atípica $(t=-1,78 ; P=0,09)$. En ambos grupos la media de semejanza al endogrupo es mayor que la de semejanza al exogrupo.

\section{Discusión}

En este trabajo hemos comparado a sujetos que tienen una representación del yo, o autoconcepto de género, prototípica para su sexo según los patrones culturales, con sujetos con una representación del yo atípica para su sexo. Se han elegido variables que se consideran indicadores de la representación que los individuos tienen de las categorías hombre y mujer y variables que miden cómo evalúan a esas categorías y qué relación personal establecen con ellas.

De los resultados se puede extraer la conclusión de que la diferencia fundamental entre estos dos grupos de sujetos se establece en las distintas representaciones que estos grupos tienen de las categorías hombre y mujer. Así lo confirman los datos obtenidos en las variables de estereotipia y homogeneidad del endogrupo y del exogrupo. Por otra parte, la correlación obtenida entre estas tres variables apoya la tesis de Park y Rothbart (1982) de que la homogeneidad del exogrupo deriva, al menos en parte, de una visión estereotipada de ese grupo.

Estos dos grupos de sujetos no difieren en la valoración que tienen de su categoría sexual, y ambos valoran más a esta categoría que a la opuesta. En cuanto a la comparación de los sujetos con su propia categoría sexual y con la otra categoría, tampoco se han encontrado diferencias. Los sujetos de identidad típica y atípica se consideran más semejantes a su endogrupo que al exogrupo. Por tanto, los sujetos de identidad atípica no pierden aspectos fundamentales de la identidad social, como la evaluación más positiva de su endogrupo y mayor identificación con él que con el exogrupo.

De los datos descriptivos que aquí se han reflejado de distancia al endogrupo y al exogrupo no se pretende sacar conclusiones. No obstante, sí se observan en ellos algunas pautas que merecen destacarse como, por ejemplo, la simetría encontrada en los dos grupos de identidad de género típica. Las mujeres que se identifican con lo femenino la mayor diferencia la establecen con los hombres en rasgos masculinos, y consideran que éstos poseen menos rasgos femeninos que ellas. Este patrón es simétrico para los hombres con identidad masculina que establecen la mayor diferencia con las mujeres en rasgos femeninos, y las consideran menos masculinas que ellos.

Respecto a los dos grupos de identidad de género atípica, lo más destacable es que el grupo de hombres con identidad femenina establece la mayor diferencia con los otros hombres en rasgos masculinos y también una importante diferencia con las mujeres a las que consideran más masculinas que ellos. 


\section{Referencias}

Beauvats, C., y Spence, J. T. (1987). Gender, Prejudice, and Categorization. Sex Roles, 16, 89-100.

BEM, S. L. (1974). The measurement of psychological androgyny, Joumal of Consulting and Clinical Psychology, 42, 155-162.

Bem, S. L. (1981). Gender Schema Theory: A Cognitive Account of Sex Typing. Psychological Re view, 88, 4, 354-364.

Deaux, K. (1985). Sex and Gender. Annual Review of Psychology, 36, 49.81.

Deaux, K.; Kite, M. E., y Lewis, L. (1985). Clustering and Gender Schema: An Uncertain Link. Personality and Social Psychology Bulletin, 11, 387-397.

Edwards, V. J., y SPENCE, J. T. (1987). Gender-Related Traits, Stereotypes, and Schemata. Joumal of Personality and Social Psychology, 53, 1, 146-154.

FrÁBLE, D. E. S. (1989). Sex Typing and Gender Ideology: Two Facets of the Individual's Gender Psychology That Go Together. Joumal of Personality and Social Psychology, 56, 1, 95-108.

Gracia, E.; Mustru, G., y Escartı, A. (1988). La socialización en la familia: técnicas de disciplina en función del sexo, en J. Fernández (Coor.): Nuevas perspectivas en el desarrollo del sexo y el género. Madrid, Pirámide, 227-248.

Markus, H.; Crane, M.; Bernstein, S., y Siladi, M. (1982). Self-Schemas and Gender. Journal of Personality and Social Psychology, 42, 1, 38-50.

Martin, C. L. (1987). A ratio Measure of Sex Stereotyping. Joumal of Personality and Social Psychology, 52, 3, 489-499.

Moya, M. (1985). Identidad, roles y estereotipos de género. Psicologia General y Aplicada, 40 (3), 457-472.

Páez, D.; Villarreal, M.; Etxeberria, A., y Valencia, J. (1987). Cognición social: Esquema y función cognitiva aplicada al mundo social, en D. Páez y col.: Pensamiento, individuo y sociedad. Cognición y representación social. Madrid, Editorial Fundamentos, 71-183.

PARK, B., y RothBART, M. (1982). Perception of Out-Group Homogeneity and Levels of Social Categorization: Memory for the Subordinate Attributes of In-Group Members. Joumal of Personality and Social Psychology, 42, 6, 1.051-1.068.

Payne, T. J.; ConNor, J. M., y Colleti, G. (1987). Gender-Based Schematic Processing: An Empirical Investigation and Reevaluation. Joumal of Personality and Social Psychology, 52, 5, 937-945.

SHERIF, C. W. (1982). Needed Conceps in the Study of Gender Identity. Psychology of Women Quarterly, 6, 375-398.

SPENCE,J. T. (1985). Gender Identity and its Implications for Concepts of Masculinity and Femininity. En T. B. Sonderegger (ed.): Nebraska Symposium on Motivation: Psychology and Gender, Lincoln, Uni. Nebraska Press, vol. 32, 59-96.

Spence, J. T.; Helmreich, R. L., y Stapp, J. (1974): The Personal Attributes Questionnaire: A measure of sex role stereotypes and masculinity-feminity. ISAS Catalog of Selected Documents in Psychology, 4, 43, n. 617 .

TAYLOR, S. E., y FALCONE, H. (1982). Cognitive Bases of Stereotyping. The Relationship Between Categorization and Prejudice. Personality and Social Psychology Bulletin, 8, 3, 426-432.

TURNER, J. C. (1987). Rediscovering the Social Group, Oxford, Blackwell (existe versión castellana en Editorial Morata, Madrid, 1989).

VEGA, M. de (1984). Introducción a la Psicología Cognitiva, Madrid, Alianza Editorial.

WILluams, J. E., y Best, D. L. (1982). Measuring Sex Stereotypes: A Thirty-Nation Study. Beverly Hills, Sage. 